\title{
Ciprofloxacin prophylaxis during autologous stem cell transplantation for multiple myeloma in patients with a high rate of fluoroquinolone-resistant gram-negative bacteria colonization
}

\author{
Alzbeta Zavrelova ${ }^{\mathrm{a}}$, Pavla Paterova ${ }^{\mathrm{b}}$, Filip Gabalec ${ }^{\mathrm{a}}$, Pavel Zak ${ }^{\mathrm{a}}$, Jakub Radocha ${ }^{\mathrm{a}}$
}

\begin{abstract}
Background. Ciprofloxacin prophylaxis used to be a standard precaution during autologous stem cell transplantation. Its benefit, with a high prevalence of fluoroqinolone resistance in the population, has recently been under scrutiny. Objective. To evaluate the impact of cessation of ciprofloxacin prophylaxis during stem cell transplantation for multiple myeloma.

Patients and Methods. Data from 104 patients with multiple myeloma transplanted during the period from January 2013 to April 2015 were retrospectively reviewed. 67 received standard ciprofloxacin prophylaxis (group A) and 37 received no antibacterial prophylaxis (group B).

Results. Febrile episodes during neutropenia, bloodstream infection (BSI) and mortality in these two cohorts were evaluated. Gram negative BSI was assessed for the colonization of quinolone-resistant gram-negative pathogens. Secondary Clostridium difficile enterocolitis presence was determined in both cohorts. There were 42 (63\%), 7 (10\%), and 0 febrile episodes, BSI and gram-negative BSI respectively in group A, and $34(92 \%), 12(32 \%)$, and $4(11 \%)$ respectively in group $B$. The differences in the number of febrile episodes $(P=0.0011)$ and deaths $(P=0.0427)$ were statistically significance. Mortality was 0 and $3(8 \%)$ in group A and group B, respectively. There was no significant difference in colonization with quinolone-resistant gram negative pathogens (25 (37\%) versus 11 (30\%)) between groups. The occurrence of Clostridium difficile colitis was the same in both groups.

Conclusion. We resumed ciprofloxacin prophylaxis for the following reasons. There was a significant reduction in febrile episodes, and consequently a sparing effect of antibiotics used for treatment of this condition. No difference in Clostridium difficile colitis occurred and the mortality rate of $8 \%$ in group B was unacceptably high.
\end{abstract}

Key words: neutropenia, autologous transplantation, multiple myeloma, ciprofloxacin prophylaxis

Received: March 28, 2018; Accepted with revision: August 16, 2018; Available online: September 7, 2018 https://doi.org/10.5507/bp.2018.047

${ }^{a} 4^{\text {th }}$ Department of Internal Medicine - Haematology, University Hospital Hradec Kralove, Charles University in Prague, Hradec Kralove, Czech Republic

'Institute of Clinical Microbiology, University Hospital Hradec Kralove, Charles University in Prague, Hradec Kralove, Czech Republic Corresponding author: Jakub Radocha, e-mail:jakub.radocha@centrum.cz

\section{INTRODUCTION}

Fluoroqinolones are widely recommended and used for prophylaxis of bacterial infection during the period of high risk neutropenia ${ }^{1,2}$. Their efficacy for hematooncology patients with neutropenia has been repeatedly proven during recent years, especially in the case of autologous stem cell transplantation ${ }^{3,4}$. Traditionally, they have served as an efficient tool for decreasing infection during neutropenia, mainly gram negative infection ${ }^{5-7}$. Not only have a reduction in febrile neutropenia and infection during neutropenia been proven, but even an impact on mortality has been shown in a study by Gafter-Gvili and Reuter $^{8,9}$. A major concern of its efficacy has been raised during recent years with the wide emergence of quinoloneresistant bacteria and a rise in breakthrough infection due to this organism ${ }^{10-13}$. Only scarce and not exact data exist concerning quinolone-resistance and its efficacy in prevention. Some investigators have linked the reduced efficacy of quinolone prophylaxis to 20 to $30 \%$ colonization by resistant pathogens ${ }^{14,15}$. Since the resistance rate for quinolones at our institution has been rising during this time (our own data), we decided to avoid their use in some indications and started with the patient undergoing stem cell transplantation for multiple myeloma. This group of patients was carefully chosen for its consistency, relatively low risk of fatal infection and short duration of neutropenia. Here we describe our observation of quinolone cessation during autologous stem cell transplantation for multiple myeloma in comparison with the rate of quinolone-resistant bacterial colonisation.

\section{PATIENTS AND METHODS}

A retrospective analysis of our common clinical practice in-patients consecutively transplanted for multiple myeloma was conducted in patients undergoing autologous stem cell transplantation for multiple myeloma at our institution during the period from January 2013 to April 2015. All patients were screened for resistant pathogen upon admission. Screening specimens consisted of rectal, nasal, 
laryngeal swab and urine cultivation. Patients' charts were then reviewed for the occurrence of febrile episode, clinical infection, bloodstream infection, Clostridium colitis occurrence, clinical outcome and antibiotic consumption as treatment. Common clinical practice of febrile patients (defined as temperature $38^{\circ} \mathrm{C}$ in two measurements at least one hour apart) included screening for infection by clinical and radiological examination, blood cultures and any other microbiologically relevant specimens. Febrile episode was counted if the patient developed fever during neutropenia after transplantation, independently of the reason for the febrile state. Blood stream infection (BSI) was counted when any bacterium was isolated from blood culture. Clostridium colitis was considered in the case of diarrhea with positive Clostridium difficile antigen and positivity of toxin A or B or confirmation by PCR technique.

All gram negative bacteria were identified by mass spectrometry using Maldi TOF (Bruker Daltonics, Germany) and antimicrobial susceptibility testing was performed by EUCAST disc diffusion method. Results were interpreted according to EUCAST criteria. If any of these materials contained gram negative bacteria not susceptible to ciprofloxacin, the patient was considered to be colonized. Detection of antigen and toxins A/B of Clostridium difficile from feces was performed using

Table 1. Demographic data.

\begin{tabular}{|c|c|c|c|c|c|}
\hline & $\begin{array}{c}\text { Group A } \\
n=67\end{array}$ & $\%$ & $\begin{array}{c}\text { Group B } \\
n=37\end{array}$ & $\%$ & $P$ \\
\hline Gender & & & & & 0.3124 \\
\hline Males & 38 & 57 & 17 & 46 & \\
\hline Females & 29 & 43 & 20 & 54 & \\
\hline Age (years) & $36-73$ & 60 mean & $33-69$ & 60 mean & 0.4445 \\
\hline Myeloma stage (Durie-Salmon) & & & & & 0.5877 \\
\hline I & 3 & 4 & 1 & 3 & \\
\hline II & 23 & 34 & 10 & 27 & \\
\hline III & 41 & 61 & 26 & 70 & \\
\hline Renal impairment & & & & & 0.3644 \\
\hline A & 51 & 76 & 25 & 68 & \\
\hline $\mathrm{B}$ & 16 & 24 & 12 & 32 & \\
\hline M-protein type & & & & & 0.6147 \\
\hline IgG kappa & 27 & 40 & 9 & 24 & \\
\hline IgG lambda & 13 & 19 & 8 & 22 & \\
\hline IgA kappa & 8 & 12 & 7 & 19 & \\
\hline IgA lambda & 5 & 7 & 2 & 5 & \\
\hline kappa free & 10 & 15 & 6 & 16 & \\
\hline lambda free & 2 & 3 & 3 & 8 & \\
\hline Other & 2 & 3 & 2 & 5 & \\
\hline Conditioning regimen & & & & & 0.0600 \\
\hline Mel 140 & 5 & 7 & 8 & 22 & \\
\hline Mel 200 & 62 & 93 & 29 & 78 & \\
\hline Number of transplants & & & & & 0.9759 \\
\hline 1st autologous & 52 & 78 & 29 & 78 & \\
\hline 2nd autologous & 13 & 19 & 8 & 22 & \\
\hline 3rd autologous & 2 & 3 & 0 & 0 & \\
\hline Disease status at transplant & & & & & 0.7239 \\
\hline $\mathrm{CR}$ & 12 & 18 & 9 & 24 & \\
\hline VGPR & 22 & 33 & 10 & 27 & \\
\hline PR & 28 & 42 & 14 & 38 & \\
\hline MR & 1 & 1 & 1 & 3 & \\
\hline SD & 0 & 0 & 1 & 3 & \\
\hline PD & 4 & 6 & 2 & 5 & \\
\hline
\end{tabular}

Mel - melphalan, CR - complete remission, VGPR - very good partial remission, PR - partial remission, MR - minimal response, SD - stable disease, PD - progressive disease 
C. diff Quik Chek Complete ${ }^{\circledR}$ (Techlab Alere, US). Positive results were confirmed by PCR detection using GeneXpert ${ }^{\circledR}$ C. difficile (Cepheid, US). Statistical evaluation was performed using MedCalc v 9.5.2.0 software (MedCalc Software, Belgium). Fisher's exact test was used for comparison of categorical variables, and t-test was used for continuous variables. All variables were considered statically significant at $P<0.05$.

Table 2. Identified bloodstream infection species.

\begin{tabular}{lcc}
\hline Species & Group A & Group B \\
\hline Staphylococcus aureus & 0 & 2 \\
Coagulase negative staphylococcus & 4 & 4 \\
Streptococcus viridans group & 1 & 1 \\
Escherichia coli & 0 & 2 \\
Enterococcus & 1 & 0 \\
Klebsiella pneumoniae & 0 & 1 \\
Pseudomonas aeruginosa & 0 & 1 \\
Listeria monocytogenes & 0 & 1 \\
Anaerobes & 1 & 2 \\
\hline
\end{tabular}

\section{RESULTS}

104 patients fulfilling selection criteria were identified. All patients received autologous stem cell transplantation for multiple myeloma. Chemotherapy consisted of melphalan $200 \mathrm{mg} / \mathrm{m}^{2}$ in 91 patients and $140 \mathrm{mg} / \mathrm{m}^{2}$ in the remaining 13 patients. Most patients underwent their first autologous transplantation, but there was 21 second and two third transplantations as well. Complete demographic data are in Table 1.

67 patients received prophylaxis with ciprofloxacin $500 \mathrm{mg}$ twice daily from the onset of chemotherapy and 37 patients received no antibacterial prophylaxis. Patients with quinolone prophylaxis were defined as group A and without prophylaxis as group B.

Colonization with ciprofloxacin-resistant gram negative bacteria was present in 25 of 67 patients (37\%) and in 11 from 37 patients (30\%) in group A and group B, respectively.

In group A 42 febrile episodes (63\%), 7 BSI (10\%) and no gram negative BSI were found. 34 patients in group B experienced febrile episodes (92\%), 12 BSI (32\%) and 4 gram negative BSI (11\%). One polymicrobial BSI was present in group B. The percentage of febrile episodes in group A was the same for colonized and non-colonized

Table 3. Antibiotic treatment.

\begin{tabular}{|c|c|c|c|c|c|}
\hline & \multicolumn{2}{|c|}{ Group A } & \multicolumn{2}{|c|}{ Group B } & \multirow{2}{*}{$P$} \\
\hline & $\mathrm{n}=67$ & $\%$ & $\mathrm{n}=37$ & $\%$ & \\
\hline Any antibiotic needed & 42 & 63 & 34 & 92 & 0.0011 \\
\hline Combination treatment necessary & 8 & 12 & 7 & 19 & 0.3872 \\
\hline \multicolumn{6}{|l|}{ Specific antibiotic used upfront } \\
\hline Piperacillin/Tazobactam & 37 & 55 & 26 & 70 & 0.1481 \\
\hline Meropenem & 3 & 4 & 7 & 19 & 0.0328 \\
\hline Vancomycin & 5 & 7 & 4 & 11 & 0.7178 \\
\hline Ceftazidime & 1 & 1 & 1 & 3 & 1.0000 \\
\hline Amoxicillin/clavulanate & 1 & 1 & 0 & 0 & 1.0000 \\
\hline Amikacin & 1 & 1 & 2 & 5 & 0.2877 \\
\hline Levofloxacin & 2 & 3 & 0 & 0 & 0.5372 \\
\hline Metronidazole & 0 & 0 & 1 & 3 & 0.3558 \\
\hline Linezolid & 0 & 0 & 1 & 3 & 0.3558 \\
\hline
\end{tabular}

Table 4. Characteristics of patients with fatal outcome.

\begin{tabular}{llll}
\hline & Patient 1 & Patient 2 & Patient 3 \\
\hline Gender & Female & Male & Male \\
Age (years) & 69 & 58 & 69 \\
Line of treatment & 1 & 2 & 2 \\
Transplantation (No.) & 1 st & 2 nd & 2nd \\
Remission status at SCT & CR & PD & VGPR \\
Myeloma type & IgA lambda & lambda free & IgG lambda \\
Myeloma stage (D-S) & III B & II A & II A \\
HCT-CI & 2 & 2 & 0 \\
Comorbidities & Arterial hypertension & Arterial hypertension & Arterial hypertension \\
Cause of death & Septic shock E. coli, & Septic shock Staph. aureus, & bilateral pneumonia, \\
& respiratory failure & multiorgan failure & agent not identified \\
\hline
\end{tabular}

D-S - Durie-Salmon, HCT-CI - Hematopoietic cell transplantation comorbidity index, CR - complete remission, PD - progressive disease, VGPR - very good partial remission 
Table 5. Overall incidence of fever, BSI, Clostridium colitis and death.

\begin{tabular}{|c|c|c|c|c|c|}
\hline & \multicolumn{2}{|c|}{ Group A } & \multicolumn{2}{|c|}{ Group B } & \multirow{2}{*}{$P$} \\
\hline & $\mathrm{n}=67$ & $\%$ & $\mathrm{n}=37$ & $\%$ & \\
\hline Febrile episode & 42 & 63 & 34 & 92 & 0.0011 \\
\hline Bloodstream infection & 7 & 10 & 12 & 32 & 0.0734 \\
\hline Clostridium enterocolitis & 4 & 6 & 3 & 8 & 0.6972 \\
\hline Death & 0 & 0 & 3 & 8 & 0.0427 \\
\hline
\end{tabular}

patients, $56 \%$ and $67 \%$ respectively. Antibiotic treatment was instituted in 42 patients (63\%) and 34 patients (92\%) in group A and B respectively. This was highly statistically significant $(P=0.0011)$. The list of identified BSI pathogens is shown in Table 2 and the list of antibiotics for treatment is shown in Table 3. First line empirical antibiotic treatment was chosen according to the clinical condition of the patient, and combination strategy was given only to the most severely compromised patients.

During this period 3 patients died and infection was the cause of death in all of them. One patient experienced septic shock caused by E. coli resistant to ciprofloxacin, and one patient died from multiorgan failure, his blood cultures having revealed staphylococcus aureus. The third patient died of pneumonia of unknown origin. All these patients were in group B. This difference was statistically significant $(P=0.0427)$. Details of these patients are shown in Table 4. No difference in Clostridium colitis diarrhea was found. It was present in 7 patients, 4 patients (6\%) in group A and 3 patients (8\%) in group B (Table 5).

\section{DISCUSSION}

After years of standard prophylaxis with ciprofloxacin during autologous stem cell transplantation for multiple myeloma at our institution we were anxious to know if this approach is still rational. Our concern resulted from the high rate of quinolone-resistant bacteria at our institution, which reached $59 \%$ for Klebsiella in 2015. We decided to stop prophylaxis after some trials showed that it was safe to omit antibiotics in neutropenic patients undergoing chemotherapy. No bacteremia-associated death was found in the study conducted by Kjellander in patients undergoing autologous stem cell transplantation with no antibacterial prophylaxis, and the same mortality rate was found in the two studies by Gudiol and Gomez with or without antibacterial prophylaxis during neutropenia ${ }^{16-18}$.

The colonisation rate with quinolone-resistant bacteria in our cohort was relatively high, up to $37 \%$. This rate is in concordance with our hospital colonization rate. This might be caused by high environmental load of resistant bacteria, especially ESBL (extended spectrum beta lactamase) producing strains, as for example surface waters around Hradec Králové are rich in ESBL-producing strains (our own surveillance, unpublished). Despite this rate we registered significantly more febrile episodes in group B and there was a clear trend for more BSI and gram negative BSI in group B also.
Consistently with work by Hamadah et al. the rates of BSI with or without prophylaxis was very much comparable in the autologous stem cell transplantation setting. The rates of BSI in the study were $24.4 \%$ and $8.6 \%$ without and with antibacterial prophylaxis, respectively ${ }^{4}$. On the other hand Gomez et al. described the same rate of BSI in patients during neutropenia with or without ciprofloxacin prophylaxis $\left(28 \%\right.$ to $26 \%$ ) (ref. $\left.{ }^{18}\right)$. These were patients treated for acute leukemia, and a colonisation rate of $24 \%$ with quinolone-resistant bacteria was dated for their institution. The reason for this inconsistent result might lie in the patient population and also the prolonged neutropenia in acute leukemia patients. During autologous transplantation for multiple myeloma the duration of neutropenia is usually shorter than after most intensive chemotherapy for other hematological malignancies. Thus there is less selection pressure in quinolone prophylaxis and a shorter time for development of breakthrough infection.

We compared the ratio of quinolone-resistant bacteria colonization in these two groups and did not find any difference. We were unable to confirm a higher rate of bloodstream infection due to quinolone-resistant bacteria on prophylaxis, as was published by Liss et al. and by Saito et al. There was no gram negative BSI in our group A (ref. ${ }^{5,19}$ ). This could also be caused by a different patient composition and usually very short hospital stay for myeloma patients undergoing autologous stem cell transplantation, as the longer stay at hospital was confirmed to be a risk factor for the development of infection with multidrug resistant gram negative bacteria ${ }^{20}$. We did not find any difference in the rate of febrile episodes within group A whether they were colonized or not. This is a quite surprising result and we did not find any consistent support for this observation in the literature.

The proportion of broad spectrum antibiotic treatment for febrile episode was much higher in group B in this analysis. Hence the impact of sparing patients from antibiotic exposure is counterbalanced by a higher consumption of much broader-spectrum antibiotics used for treatment of febrile episode. Quinolone exposure is believed to increase the risk of Clostridium difficile colitis ${ }^{21}$. Similar rates were noted in our study in these two cohorts, which is consistent with data in hematological patients from Simondsen ${ }^{22}$.

During previous years with quinolone prophylaxis the mortality of multiple myeloma patients during autologous transplantation at our institution was below 1\%, similar to what was reported by Gertz and Papanikolaou ${ }^{23,24}$. Our mortality rate of $8 \%$ in group B was alarming and above all 
it was clearly related to infection episode. We might speculate that in one case of septic shock caused by quinoloneresistant $E$. coli, prophylaxis with ciprofloxacin would not have changed the clinical course of sepsis, but in reality we cannot rule out a clinical impact of ciprofloxacin exposure, which might mitigate speed and severity of septic shock development. This led us to return to our previous policy and restart quinolone prophylaxis. We observed no more fatalities after prophylaxis had been reinstituted on the subsequent cohort of patients, and the evaluation of the data is still ongoing. We conclude that there is a benefit of quinolone prophylaxis in myeloma patients undergoing ASCT.

Acknowledgement: Supported by PROGRES Q40/8 and by MH CZ - DRO (UHHK, 00179906). The authors are grateful to Ian McColl MD, PhD for assistance with the manuscript.

Authors contributions: AZ, JR: manuscript writing, collected and reviewed clinical data; PP: manuscript writing, collected and reviewed microbiological and susceptibility data; FG, PZ: reviewed and approved the manuscript.

Conflict of interest statement: None declared.

\section{REFERENCES}

1. Flowers CR, Seidenfeld J, Bow EJ, Karten C, Gleason C, Hawley DK, Kuderer NM, Langston AA, Marr KA, Rolston KV, Ramsey SD. Antimicrobial prophylaxis and outpatient management of fever and neutropenia in adults treated for malignancy: American Society of Clinical Oncology clinical practice guideline. J Clin Onco 2013;31(6):794-810.

2. Bow EJ. Point: fluoroquinolone-based antibacterial chemoprophylaxis in neutropenic cancer patients works for defined outcomes in defined populations, but must be used wisely. J Natl Compr Cancer Netw 2004;2(5):433-44.

3. Hong J, Moon SM, Ahn HK, Sym SJ, Park YS, Park J, Cho YK, Cho EK, Shin DB, Lee JH. Comparison of characteristics of bacterial bloodstream infection between adult patients with allogeneic and autologous hematopoietic stem cell transplantation. Biol Blood Marrow Transplant 2013;19(6):994-9.

4. Hamadah A, Schreiber Y, Toye B, McDiarmid S, Huebsch L, Bredeson $C$, Tay J. The use of intravenous antibiotics at the onset of neutropenia in patients receiving outpatient-based hematopoietic stem cell transplants. PloS One 2012;7(9):e46220.

5. Saito T, Yoshioka S, linuma Y, Takakura S, Fujihara N, Ichinohe T, Ishikawa T, Uchiyama T, Ichiyama S. Effects on spectrum and susceptibility patterns of isolates causing bloodstream infection by restriction of fluoroquinolone prophylaxis in a hematology-oncology unit. Eur J Clin Microbiol Infect Dis 2008;27(3):209-16.

6. Kern WV, Klose K, Jellen-Ritter AS, Oethinger M, Bohnert J, Kern $\mathrm{P}$, Reuter S, von Baum H, Marre R. Fluoroquinolone resistance of Escherichia coli at a cancer center: epidemiologic evolution and effects of discontinuing prophylactic fluoroquinolone use in neutropenic patients with leukemia. Eur J Clin Microbiol Infect Dis 2005;24(2):111-8.

7. Bucaneve G, Micozzi A, Menichetti F, Martino P, Dionisi MS, Martinelli G, Allione B, D'Antonio D, Buelli M, Nosari AM, Cilloni D, Zuffa E, Cantaffa R, Specchia G, Amadori S, Fabbiano F, Deliliers GL, Lauria F, Foà R, Del Favero A; Gruppo Italiano Malattie Ematologiche dell'Adulto (GIMEMA) Infection Program. Levofloxacin to prevent bacterial infection in patients with cancer and neutropenia. N Engl J Med 2005;353(10):977-87.

8. Gafter-Gvili A, Fraser A, Paul M, Leibovici L. Meta-analysis: antibiotic prophylaxis reduces mortality in neutropenic patients. Ann Intern Med 2005;142(12):979-95.
9. Reuter S, Kern WV, Sigge A, Döhner H, Marre R, Kern P, von Baum H. Impact of fluoroquinolone prophylaxis on reduced infection-related mortality among patients with neutropenia and hematologic malignancies. Clin Infect Dis 2005;40(8):1087-93.

10. Mikulska M, Viscoli C, Orasch C, Livermore DM, Averbuch D, Cordonnier C, Akova M7; Fourth European Conference on Infections in Leukemia Group (ECIL-4), a joint venture of EBMT, EORTC, ICHS, ELN and ESGICH/ESCMID. Aetiology and resistance in bacteraemias among adult and paediatric haematology and cancer patients. J Infect 2014;68(4):321-31.

11. Trecarichi EM, Tumbarello M. Antimicrobial-resistant Gram-negative bacteria in febrile neutropenic patients with cancer: current epidemiology and clinical impact. Curr Opin Infect Dis 2014;27(2):200-10.

12. Montassier E, Batard E, Gastinne T, Potel G, de La Cochetière MF. Recent changes in bacteremia in patients with cancer: a systematic review of epidemiology and antibiotic resistance. Eur J Clin Microbiol Infect Dis 2013;32(7):841-50.

13. Schelenz $S$, Nwaka $D$, Hunter PR. Longitudinal surveillance of bacteraemia in haematology and oncology patients at a UK cancer centre and the impact of ciprofloxacin use on antimicrobial resistance. J Antimicrob Chemother 2013;68(6):1431-8.

14. Ng ES-T, Liew Y, Earnest A, Koh LP, Lim S-W, Hsu LY. Audit of fluoroquinolone prophylaxis against chemotherapy-induced febrile neutropenia in a hospital with highly prevalent fluoroquinolone resistance. Leuk Lymphoma 2011;52(1):131-3.

15. Bow EJ. Fluoroquinolones, antimicrobial resistance and neutropenic cancer patients. Curr Opin Infect Dis 2011;24(6):545-53.

16. Kjellander C, Björkholm M, Cherif H, Kalin M, Giske CG. Hematological: Low all-cause mortality and low occurrence of antimicrobial resistance in hematological patients with bacteremia receiving no antibacterial prophylaxis: a single-center study. Eur J Haematol 2012;88(5):422-30.

17. Gudiol C, Bodro M, Simonetti A, Tubau F, González-Barca E, Cisnal M, Domingo-Domenech $\mathrm{E}$, Jiménez L, Carratalà J. Changing aetiology, clinical features, antimicrobial resistance, and outcomes of bloodstream infection in neutropenic cancer patients. Clin Microbiol Infect 2013;19(5):474-9

18. Gomez L, Garau J, Estrada C, Marquez M, Dalmau D, Xercavins M Martí JM, Estany C. Ciprofloxacin prophylaxis in patients with acute leukemia and granulocytopenia in an area with a high prevalence of ciprofloxacin-resistant Escherichia coli. Cancer 2003;97(2):419-24.

19. Liss BJ, Vehreschild JJ, Cornely OA, Hallek M, Fätkenheuer G, Wisplinghoff $H$, Seifert $H$, Vehreschild MJ. Intestinal colonisation and blood stream infections due to vancomycin-resistant enterococci (VRE) and extended-spectrum beta-lactamase-producing Enterobacteriaceae (ESBLE) in patients with haematological and oncological malignancies. Infection 2012;40(6):613-9.

20. Garnica M, Maiolino A, Nucci M. Factors associated with bacteremia due to multidrug-resistant Gram-negative bacilli in hematopoietic stem cell transplant recipients. Braz J Med Biol Res 2009;42(3):28993.

21. McCusker ME, Harris AD, Perencevich EN, Roghmann MC. Fluoroquinolone Use and Clostridium difficile-Associated Diarrhea. Emerg Infect Dis 2003; 9(6):730-3.

22. Simondsen KA, Reed MP, Mably MS, Zhang Y, Longo WL. Retrospective analysis of fluoroquinolone prophylaxis in patients undergoing allogeneic hematopoietic stem cell transplantation. J Oncol Pharm Pract 2013;19(4):291-7.

23. Gertz MA, Ansell SM, Dingli D, Dispenzieri A, Buadi FK, Elliott MA, Gastineau DA, Hayman SR, Hogan WJ, Inwards DJ, Johnston PB, Kumar S, Lacy MQ, Leung N, Micallef IN, Porrata LF, Schafer BA, Wolf $\mathrm{RC}$, Litzow MR. Autologous stem cell transplant in 716 patients with multiple myeloma: low treatment-related mortality, feasibility of outpatient transplant, and effect of a multidisciplinary quality initiative. Mayo Clin Proc 2008;83(10):1131-8.

24. Papanikolaou X, Maltezas D, Repousis P, Athanassopoulos A, Alexia S, Megalakaki K, Kotsopoulou M, Mitsouli-Mentzikof C. High dose chemotherapy and autologous stem cell transplantation in patients with multiple myeloma: the experience of a single haematological unit. J BUON 2008;13(2):193-7. 